\title{
Dual Tree Wavelet based OFDM: A Performance Calculation of Bit Error Rate
}

\author{
Nemir Al-Azzawi \\ Al Khwarizmi College of Engineering, University of Baghdad, Iraq
}

\begin{abstract}
In the rapid development of digital wireless communications, have increased demands for wireless systems with high efficiency performance. In this paper, an efficient OFDM system has been proposed based on dual-tree complex wavelet transform (DT-CWT). The mathematics behind the proposed OFDM system is explained. Moreover, comparative study has been made between the traditional OFDM and the OFDM based on DT-CWT. The proposed scheme achieves excellent improvements in bit error rate (BER) over conventional OFDM and wavelet packet modulation (WPM) systems. The proposed technique gives a significant improvement in Bit Error Rate (BER) performance in Additive White Gaussian Noise (AWGN) channels, flat fading channels (FFC), and multi-path selective fading channels (SFC) compared with traditional techniques. The simulation results performance is described in BER as a function of Signal to Noise Ratio (SNR).
\end{abstract}

\section{Keywords}

OFDM; Wavelet; DWT; WPT; DT-CWT; FFT; Multicarrier Modulation; BER.

\section{INTRODUCTION}

The orthogonal frequency division multiplex (OFDM), the modulation concept being used for many wireless and radio communications radio applications from DAB, DVB, Wi-Fi and Mobile Video. OFDM is a multi-carrier transmission technique, which divides the available spectrum into many carriers, each one being modulated by a low rate data stream. The modulation technique works by simultaneously transmitting $N$ data symbols through $N$ carriers, thus reducing the symbol rate to one $N^{\text {th }}$ of the original symbol rate. Also it increases the symbol duration by $N$ times. The technique offers more robust against inter-symbol interference (ISI) caused by channel dispersions and multipath interference. Nowadays, OFDM has been acknowledged as the standard in several wired line and wireless applications such as the European Digital Audio Broadcasting (DAB), terrestrial digital videobroadcast (DVB-T) systems, asynchronous digital subscriber line (ADSL) and WiMAX (IEEE 802. 16) [1]. A new networking approach based on IP/optical OFDM technologies is proposed by [2], providing an adaptive mechanism of bandwidth provisioning and pipe resizing for dynamic traffic flows. OFDM has several advantages compared to other type of modulation techniques such as bandwidth efficiency, overcome the effect of ISI, and combats the effect of frequency selective fading and burst error[1]. Nevertheless, the OFDM system has some drawback such as the high which means that the linear amplifier has to have a large dynamic range to avoid distorting the peaks. The other limitation of OFDM in many applications is that it is very sensitive to frequency errors caused by frequency differences between the local oscillators in the transmitter and the receiver [3-4].
In wireless communication reception, the reliability of Fast Fourier-based (OFDM-FFT) is limited because of the timevarying nature of the channel. This induces intercarrier interference (ICI) and increases inaccuracies in channel tracking. This can be avoided effectively at the cost of power loss and bandwidth expansion by inserting a cyclic prefix (CP) guard interval before each block of parallel data symbols. However, this reduces the spectral efficiency [1]. The Haarorthonormal wavelets, discrete wavelet-based OFDM (OFDMDWT) was found to decrease the intersymbol interference (ISI) and ICI, which are caused by the loss in orthogonality between the carriers[5]. OFDM-DWT be able to too maintain much higher spectrum efficiency than OFDM-FFT to represent data modulation and demodulation the concept of scalar wavelets has been exploited as wavelet modulation, for multi rate transmissions [5-6]. The OFDM-FFT uses complex exponential bases functions, and was replaced with orthonormal wavelets to reduce the level of interference. Farhang et al. proposed multi-carrier modulations that with every data packet made up of a number of complex-valued sinusoids that are modulated through information symbols [7]. Manglani and Bell showed in simulation results that using wavelet-based OFDM is the superior spectral control properties of wavelet filters over Fourier filters [8]. The SNR measure can be successfully increased and achieves considerably lower bit error rates and performs better than OFDM-FFT when using the wavelet OFDM within a desired wavelet basis function[6, 8-10].

Wavelet transforms have been considered as alternative platforms for replacing IFFT and FFT [11-13]. By using the wavelet transform, the spectral containment of the channels is better since it does not use CP [14]. It employs Low Pass Filter (LPF) and High Pass Filter (HPF) operating as Quadrature Mirror Filters satisfying perfect reconstruction and orthonormal bases properties. The transform uses filter coefficients as approximate and detail in LPF and HPF respectively. The approximated coefficients is sometimes referred to as scaling coefficients, whereas, the detailed is referred to wavelet coefficients [15]. The performance comparison of original OFDM and wavelet for several multipath wireless channels is presented [16-17]. The wavelet packet modulation (WPM) system has an advanced spectral efficiency though providing robustness with regard to interchannel interference than the conventional OFDM system, because of low out-of-band energy (low sidelobes) [18]. N.G. Kingsbury and Selesnick [19-21] develop dual-tree complex wavelet transform (DT-CWT) in image processing application. It maintains the desirability for feature formation such as small feature vector in comparison with Gabor wavelet, illumination invariant feature etc. In this paper, an OFDM system based on DT-CWT transform is proposed. The proposed system is simply implemented by a two scale equations. The system is compared with the traditional OFDM systems using MATLAB simulink programs. Simulation results show that the best BER performance exhibits by the OFDM-DT-CWT system. 


\section{FOURIER-BASED OFDM (OFDM- FFT)}

The input data stream is first mapped into the QAM modulation scheme according to the QAM constellation mapping. Then the complex number output is transformed from serial to parallel into N-points IFFT to generate the OFDM symbols. The output data from IFFT is now converted from parallel to serial and a cyclic prefix is added to the data. The data are sent via wireless channel after being converted to frame structure (serial data stream). The frame structure consists of the modulated data and the pilot signal which is used for channel estimation and compensation. The channel consists of a multipath fading (flat fading channel or frequency selective fading channel) with AWGN. At the receiver the reverse operations of the encoding processes are employed. The cyclic prefix is removed and a serial to parallel conversion is done for the signal. A FFT with $\mathrm{N}$ points is used to convert the signal from time to frequency domain. Then the effective channel is compensated after the OFDM demodulation, the signal demapper is used to recover the transmitted signal [22].

The system model for FFT-based OFDM will not be discussed in detail as it is well known in the literature. Thus, we simply present a brief description about it. The data generator produced $k$ in random binary form. It is first being processed by a constellation mapping. M-ary QAM modulator is used for this work to map the raw binary data to appropriate QAM symbols $X_{m}$. These symbols are then input into the IFFT block. This involves taking $\mathrm{N}$ parallel streams of QAM symbols ( $\mathrm{N}$ being the number of sub-carriers used in the transmission of the data) and performing an IFFT operation on this parallel stream. The output in discrete time domain is as follows:

$X_{k(n)}=\frac{1}{\sqrt{N}} \sum_{i=0}^{N-1} X_{m(i)} \exp \left(j 2 \pi \frac{n}{N} i\right)$

where $X_{k(n)} \mid 0 \leq n \leq N-1$ is a sequence in the discrete time domain and $X_{m(i)} \mid 0 \leq i \leq N-1$ are complex numbers in the discrete frequency domain. The cyclic prefix (CP) is lastly added before transmission to minimize the inter-symbol interference (ISI). At the receiver, the process is reversed to obtain the decoded data. The $\mathrm{CP}$ is removed to obtain the data in the discrete time domain and then processed to FFT for data recovery. The output of the FFT in the frequency domain is as follows:

$$
U_{m(i)}=\sum_{n=0}^{N-1} U_{k(n)} \exp \left(-j 2 \pi \frac{n}{N} i\right)
$$

\section{THE PROPOSED TECHNIQUE DESCRIPTION}

The Fourier Transform is possibly the most accepted transform used to obtain the frequency spectrum of a signal. But the Fourier Transform is only appropriate for signals whose frequency content does not change with time. The Fourier Transform does not tell at which time these frequency components happen. To solve this problem, the Wavelet transform, which was developed in the last two decades, provides a better time-frequency representation of the signal than any other existing transforms[5-6, 23]. Kingsbury [24], proposed dual-tree complex wavelet transform (DT-CWT) that provides shift invariance approximation. We will briefly discuss the adjustment to the dual-tree complex wavelet transform (DT-CWT), in one dimension. Extension to higher dimensions is then straight forward. The 1D DT-CWT is implemented using two parallel DWT filter banks, the first filter bank uses the real parts of the complex wavelet and scaling filters (respectively $G_{0}$ and $H_{0}$ ), while in the second filter bank, the imaginary parts of the wavelet and scaling filters (respectively $G_{l}$ and $H_{l}$ ) are applied. Finally, the output of both filter banks are mixed together. In particular, whereas wavelets have associated scaling $\varphi(t)$ and wavelet functions $\psi(t)$ , DT-CWT have two scaling and wavelet functions. For notational convenience, the set of scaling functions be able to be written using the vector notation $\Phi(t)=\left[\Phi_{1}(t) \Phi_{2}(t)\right]^{T}$. The DT-CWT function is defined from the set of wavelet functions as $\psi(t)=\left[\psi_{1}(t) \psi_{2}(t)\right]^{T}$. When $r=1, \Psi(t)$ is called a scalar wavelet, or simply wavelet. All wavelet functions $(t) \psi_{2}(t) \ldots \psi_{r}$ re in $W_{0} \subset V_{-1}$. The DT-CWT employs two real discrete wavelet transform; the upper part of the filter bank gives the real part of the transform while the lower one gives the imaginary part. The DT-CWT two scale $(r=2)$ equations resemble those for scalar wavelets[25-26]:

$$
\begin{aligned}
& \psi(t)=\sqrt{2} \sum_{k=-\infty}^{\infty} H_{k} \Phi(2 t-k), \\
& \Phi(t)=\sqrt{2} \sum_{k=-\infty}^{\infty} G_{k} \Phi(2 t-k),
\end{aligned}
$$

where $G_{k}$ is a matrix lowpass filter and $H_{k}$ is a matrix highpass filter. We will also call $G_{k}$ and $H_{k}$ multifilters. However $G_{k}$ and $H_{k}$ are matrix filters wavelets coefficients. (i. e., $G_{k}$ and $H_{k}$ are $r \times r$ matrices instead of scalar values). The matrix elements in these filters provide further degrees of freedom than a traditional scalar wavelet. The aim is to increase the number of scaling functions to raise the approximation power to more than one scaling function. In [27-28] a new OFDM system was being introduced, based on DT-CWT. It has two or more low-pass and high-pass filters. Practice the values of $G_{0}, G_{1}$ and $H_{0}, H_{1}$ are as follows [29]:

$$
G_{0}=\left[\begin{array}{cc}
\frac{3}{5 \sqrt{2}} & \frac{4}{5} \\
\frac{-1}{20} & \frac{-3}{10 \sqrt{2}}
\end{array}\right], G_{1}=\left[\begin{array}{cc}
\frac{3}{5 \sqrt{2}} & 0 \\
\frac{9}{20} & \frac{1}{\sqrt{2}}
\end{array}\right]
$$

$$
H_{0}=\frac{1}{10}\left[\begin{array}{cc}
\frac{-1}{2} & \frac{-3}{\sqrt{2}} \\
\frac{1}{\sqrt{2}} & 3
\end{array}\right], H_{1}=\frac{1}{10}\left[\begin{array}{cc}
\frac{9}{2} & \frac{-10}{\sqrt{2}} \\
\frac{-9}{\sqrt{2}} & 0
\end{array}\right]
$$

The multiscaling and DT-CWT functions are also quite smooth almost differentiable. The two-scale equations (6) and (7) can be realized as a matrix filter bank (see Figure 1) operating on input data streams and filtering them into 2 output data streams, each of which is down sampled by a factor two. If we denote by $x(t)$ a given signal and assume that $x(t) \in V_{0}$, then 
full DT-CWT decomposition of the signal $x(t)$ can be found by iterative filtering of the scaling coefficients:

$$
v_{j, k}=\sum_{m} G_{m-2 k} v_{j-1, m}, w_{j, k}=\sum_{m} H_{m-2 k} v_{j-1, m}
$$

The original signal can be reconstructed from the DT-CWT coefficients by means of the synthesis equation:

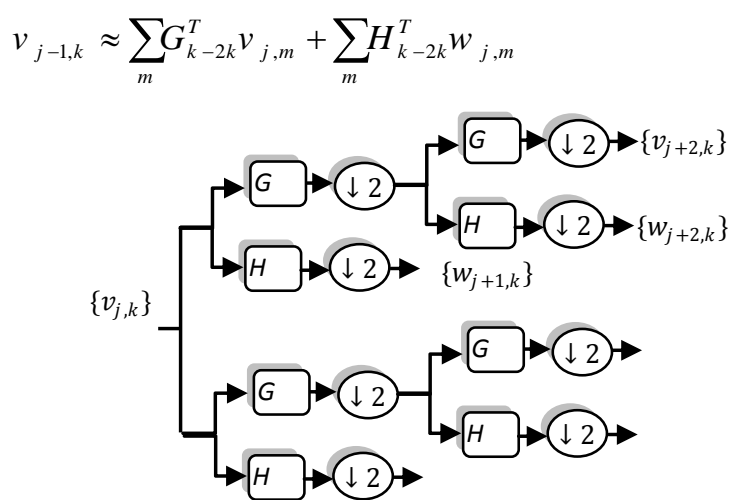

Fig 1: A DT-CWT filter bank, iterated once

In the one-dimensional signals, the computational method for DT-CWT and inverse DT-CWT by an oversampled scheme of preprocessing (repeated row), is convenient and influential and further performance gains were made by looking into alternative orthogonal bases functions and finding a better transform than Fourier and wavelet transforms.

The multifilter bank is needs two input rows. The goal of preprocessing is to relate the given scalar input signal of length $N$ to a sequence of length-2 vectors to start the analysis algorithm and reduce noise effects. Figure 2, is the schematic block diagram used for the proposed OFDM transceiver simulations. Examination input dimensions: Input vector should be of length $\mathrm{N}$, where $\mathrm{N}$ must be power of two. Constructing a N/2×N/2 transformation matrix $T$ using LPFs and HPFs matrices given in equations (4) and (5) respectively. For computing DT-CWT, scalar wavelet transform matrices can be written as follows:

$$
T=\left[\begin{array}{ccccccccccc}
H_{0} & H_{1} & 0 & 0 & 0 & 0 & \ldots & 0 & 0 & 0 & 0 \\
0 & 0 & H_{0} & H_{1} & 0 & 0 & \ldots & 0 & 0 & 0 & 0 \\
\vdots & \vdots & \vdots & \vdots & \vdots & \vdots & \ldots & \vdots & \vdots & \vdots & \vdots \\
0 & 0 & 0 & 0 & 0 & 0 & \ldots & 0 & 0 & H_{0} & H_{1} \\
G_{0} & G_{1} & 0 & 0 & \vdots & \vdots & \ldots & 0 & 0 & 0 & 0 \\
0 & 0 & G_{0} & G_{1} & 0 & 0 & \ldots & 0 & 0 & 0 & 0 \\
\vdots & \vdots & \vdots & \vdots & \vdots & \vdots & \ldots & \vdots & \vdots & \vdots & \vdots \\
0 & 0 & 0 & 0 & 0 & 0 & \ldots & G_{0} & G_{1} & 0 & 0 \\
0 & 0 & 0 & 0 & 0 & 0 & \ldots & 0 & 0 & G_{0} & G_{1}
\end{array}\right]
$$

After substituting the matrix filter coefficients values, an $\mathrm{N} \times \mathrm{N}$ transformation matrix results. Transformation of input vector which can be done by applying matrix multiplication to the $\mathrm{N} / 2 \times \mathrm{N} / 2$ constructed transformation matrix by the $\mathrm{N} \times 1$ preprocessing input vector. The pilot-assisted channel estimator is used here to combat the channel fading effects since it is an efficient method especially for the case of slow fading channels. IDT-CWT works in a similar fashion to an IFFT or IDWT [5]. It takes as the input QAM symbols and outputs them in parallel time-frequency "subcarriers".

\section{SIMULATION RESULTS AND DISCUSSION}

In this section, the OFDM-DT-CWT system behavior is studied through MATLAB simulink. In addition, comparisons between the OFDM-FFT[22], OFDM-DWT[5] systems and the proposed OFDM-DT-CWT are done in terms of BER under different channel conditions. AWGN channels, Flat Fading Channels (FFC), and frequency selective fading channels are considered during simulations. The frame consists of 64 samples each is converted into two bits per sample to be suited for QAM modulator. The IQ-Mapper converts the bits into samples and then performs QAM modulation. Afterwards, the complex data are generated which are the input to the three systems. Thereafter, the output from the OFDM modulator is transmitted through AWGN channel which adds Gaussian noise to the transmitted signals. Subsequently, the signals are received and OFDM demodulated, IQ-demapped, and converted to bits respectively. All of the transformed models in OFDM-FFT, OFDM-DWT and OFDM-DT-CWT systems used the parameters as shown in Table I. The simulation results of proposed OFDM-DT-CWT system in AWGN channel are shown in Figure 3. From which it is clearly seen that OFDMDT-CWT has much better performance than OFDM-FFT and OFDM-DWT. This reflects the fact that the orthogonality of proposed based OFDM-DT-CWT is much higher than the orthogonality of OFDM-FFT.

In a flat fading channel with AWGN all frequency components in the signal are affected by a constant attenuation and a linear phase distortion of the channel. A Doppler frequency of $10 \mathrm{~Hz}$ is used in this simulation and the results of simulations are provided in Figure 4. It can be seen from Figure 4, that OFDM-DT-CWT outperforms significantly the other system for this channel model.

\begin{tabular}{|c|c|c|c|}
\hline sretemaraP & OFDM-FFT & OFDM-DWT & $\begin{array}{l}\text { OFDM-DT- } \\
\text { CWT }\end{array}$ \\
\hline Variables & $\begin{array}{l}\text { Matrix } \\
\text { Values }\end{array}$ & Matrix Values & Matrix Values \\
\hline $\mathrm{N}$ & 64 & 64 & 64 \\
\hline ns & 1000 & 1000 & 1000 \\
\hline $\mathrm{CP}$ & 8 & 0 & 0 \\
\hline $\mathrm{d}(\mathrm{N} \times \mathrm{ns})$ & $64 \times 1000$ & $64 \times 1000$ & $64 \times 1000$ \\
\hline$X_{m}(\mathrm{~N} \times \mathrm{ns})$ & $64 \times 1000$ & $64 \times 1000$ & $64 \times 1000$ \\
\hline$X_{k}$ & $64000 \times 1$ & $64000 \times 1$ & $64000 \times 1$ \\
\hline$U_{k}$ & $64000 \times 1$ & $64000 \times 1$ & $64000 \times 1$ \\
\hline $\mathrm{U}_{\mathrm{m}}(\mathrm{N} \times \mathrm{ns})$ & $64 \times 1000$ & $64 \times 1000$ & $64 \times 1000$ \\
\hline $\mathrm{d}^{\prime}(\mathrm{N} \times \mathrm{ns})$ & $64 \times 1000$ & $64 \times 1000$ & $64 \times 1000$ \\
\hline
\end{tabular}

Table 1. Table Simulation variables and their matrix values 


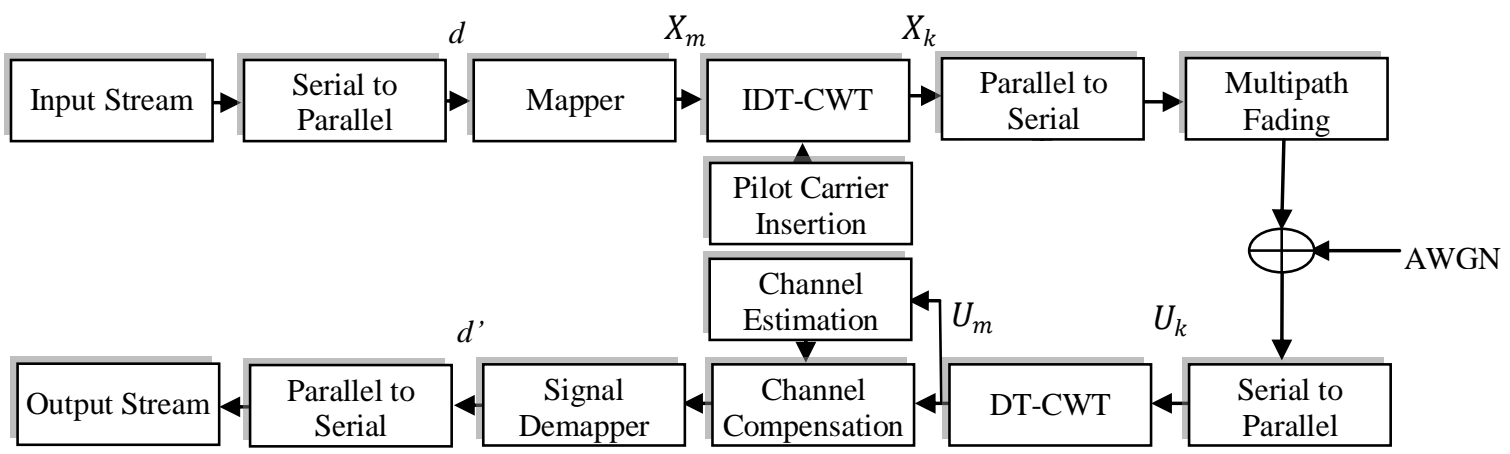

Fig. 2: Block diagram of proposed OFDM DT-CWT.

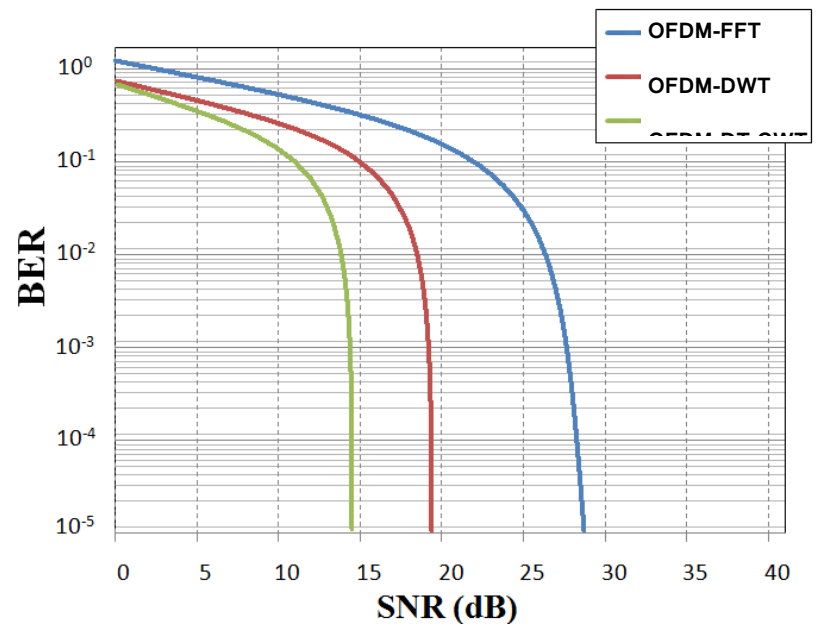

Fig. 3: BER performance in AWGN channel model.

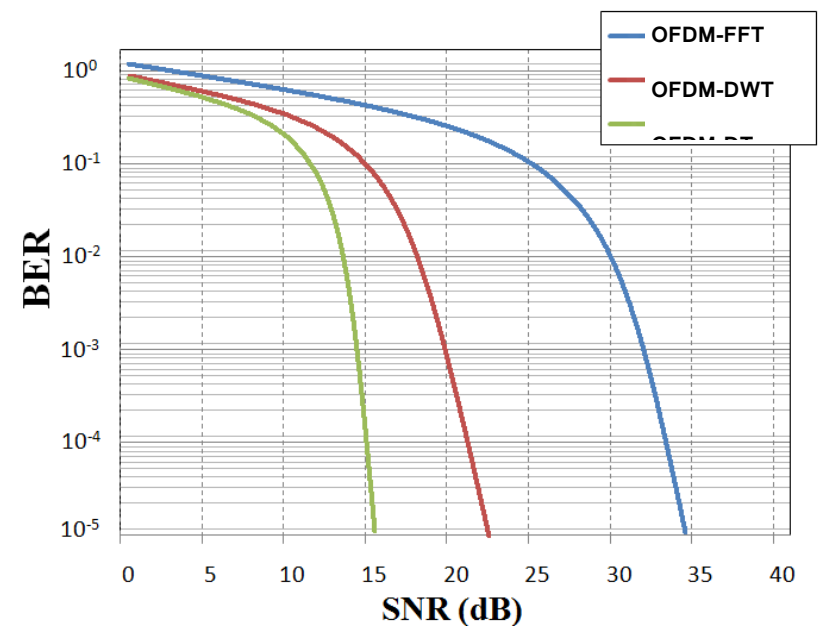

Fig, 4: BER performance at Max Doppler Shift $=10 \mathrm{~Hz}$.

In multipath frequency selective Rayleigh distributed channels with AWGN. Two rays channel is assumed here with a second path gain of $-8 \mathrm{~dB}$ at a maximum delay from the second path of $\tau_{\max }=0.1 \mu \mathrm{sec}$

for several values of SNR. Figure 5 shows simulations at maximum Doppler shift, $f_{D} .=10 \mathrm{~Hz}$.

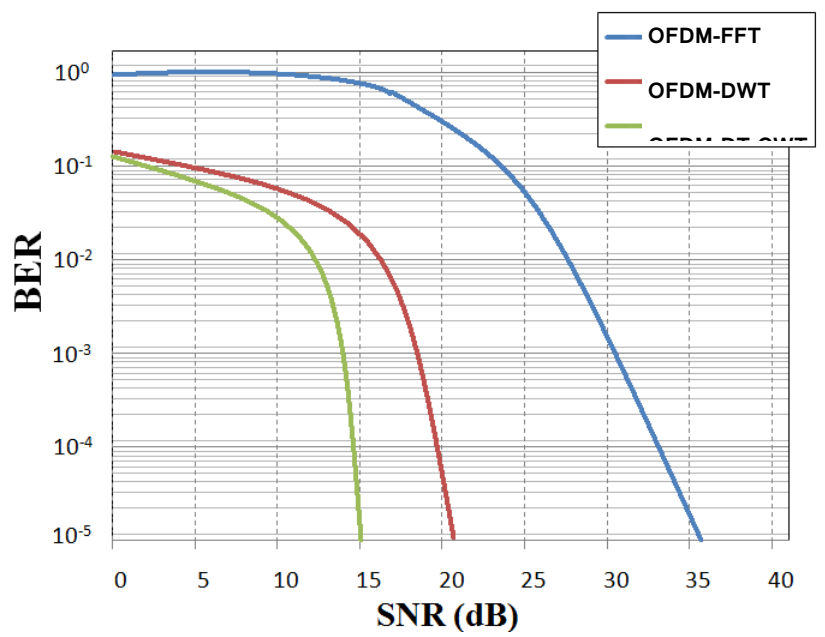

Fig. 5: The BER performance in Selective Fading Channel at Max Doppler Shift=10Hz.

It is seen that OFDM systems are very sensitive systems to the variation of Doppler frequency in selective fading channel. It is clearly seen from figures that the performance of OFDM-DTCWT is superior to that of OFDM-FFT, OFDM-DWT system.

\section{CONCLUSIONS}

In this paper, a proficient technique OFDM-DT-CWT system was proposed. Extensive simulation programs were performed to investigate the efficiency of the proposed system compared with traditional OFDM-DWT and OFDM-FFT systems. In AWGN, flat fading channel and selective fading channel the OFDM-DT-CWT outperform the other OFDM system and offers a large improvement in SNR and better performance. Also it can be concluded that the OFDM-DT-CWT is less sensitive to channel parameters variations like maximum Doppler shift in selective fading channels as compared with the standard OFDM structure. Therefore, this structure can be considered as an alternative to the conventional OFDM. Although, OFDM-FFT offers a low complexity structure than OFDM-DT-CWT, however, the use of CP reduces its spectral efficiency and wastes transmit power. The proposed OFDMDT-CWT system is robust for multipath channels.

\section{REFERENCES}

[1] A. R. S. Bahai, et al., Multi-carrier digital communications : theory and applications of OFDM, 2nd ed. New York: Springer, 2004.

[2] W. Wei, et al., "Adaptive IP/optical OFDM networking design," in Optical Fiber Communication (OFC), collocated National Fiber Optic Engineers Conference, 2010 Conference on (OFC/NFOEC), 2010, pp. 1-3. 
[3] J. G. Andrews, et al., Fundamentals of WiMAX : understanding broadband wireless networking. Upper Saddle River, NJ: Prentice Hall, 2007.

[4] Y. S. Cho, MIMO-OFDM wireless communications with MATLAB. Singapore ; Hoboken, NJ: J. Wiley \& Sons (Asia), 2010.

[5] B. G. Negash and H. Nikookar, "Wavelet-based multicarrier transmission over multipath wireless channels," Electronics Letters, vol. 36, pp. 1787-1788, 2000.

[6] G. W. Wornell and A. V. Oppenheim, "Wavelet-based representations for a class of self-similar signals with application to fractal modulation," IEEE Transactions on Information Theory, vol. 38, pp. 785-800, 1992.

[7] B. Farhang-Boroujeny and H. Moradi, "OFDM Inspired Waveforms for 5G," IEEE Communications Surveys \& Tutorials, vol. 18, pp. 2474-2492, 2016.

[8] M. J. Manglani and A. E. Bell, "Wavelet modulation in Gaussian and Rayleigh fading channels," in Proceedings of the IEEE Military Communications Conference (MILCOM '01), Electrical Engineering, Virginia Polytechnic Institute and State University, McLean, Va, USA, October, 2001.

[9] E. Lawrey, "The Suitability of OFDM as A Modulation Technique for Wireless Telecommunications, with A CDMA Comparison," thesis, James Cook University, Australia, 1997.

[10] T. J. Yew, "Multiwavelets and Scalable Video Compression," Ph.D. Thesis, Department of Electrical and Computer Engineering, National University of Singapore, Singapore, 2002

[11] R. Mirghani and M. Ghavami, "Comparison between Wavelet-based and Fourier-based Multicarrier UWB Systems," IET Communications, vol. 2, pp. 353-358, 2008.

[12] S. D. Sandberg and M. A. Tzannes, "Overlapped discrete multitone modulation for high speed copper wire communications," IEEE Journal on Selected Areas in Communications, vol. 13, pp. 1571-1585, 1995.

[13] K. Anoh, et al., "PAPR reduction of wavelet-OFDM systems using pilot symbols," in 2018 IEEE International Symposium on Power Line Communications and its Applications (ISPLC), 2018, pp. $1-6$.

[14] D. Karamehmedovic, et al., "Performance of Wavelet Packet Modulation and OFDM in the Presence of Carrier Frequency and Phase Noise," in Proceedings of the 1st European Wireless Technology Conference, EuMA, Amsterdam, Netherlands, 2008, pp. 166-169.

[15] M. Weeks, Digital signal processing using MATLAB and wavelets, 2nd ed. Sudbury, Mass.: Jones and Bartlett Publishers, 2011.

[16] U. Khan, et al., "Performance Comparison of Wavelet Packet Modulation and OFDM for Multipath Wireless
Channel," presented at the International Conference Computer, Control and Communication, Karachi, 2009.

[17] F. Farrukh, et al., "Performance comparison of DFTOFDM and Wavelet-OFDM with zero-forcing equalizer for FIR channel equalization," in Proceeding International Conference Electrical Engineering, 2000, pp. 1-5.

[18] A. Jamin and P. Mahonen, "Wavelet Packet Modulation for Wireless Communications," Journal of Wireless Communications and networking, vol. 5, pp. 123-137, Mar. 2005.

[19] I. W. Selesnick, et al., "The Dual-Tree Complex Wavelet Transform," IEEE Signal Processing Magazine, pp. 123151, 2005.

[20] N. G. Kingsbury, "Image Processing with Complex Wavelets," Philosophical Transactions of the Royal Society A: Mathematical, vol. 357, pp. 2543-2560, 1999.

[21] H. Hu, "Multiscale Illumination Normalization for Face Recognition using Dual-Tree Complex Wavelet Transform in Logarithm Domain," Computer Vision and Image Understanding, vol. 115, pp. 1384-1394, 2011.

[22] L. Hanzo, OFDM and MC-CDMA for broadband multiuser communications, WLANs, and broadcasting. Piscataway, N.J. Chichester, England ; Hoboken, NJ: IEEE Press, John Wiley, 2003.

[23] J. S. Geronimo, et al., "Fractal Functions and Wavelet Expansions Based on Several Scaling Functions," Journal of Approximation Theory, vol. 78, pp. 373-401, 1994.

[24] N. Kingsbury, "Shift invariant properties of the dual-tree complex wavelet transform," in Proceedings IEEE International Conference on Acoustics, Speech, and Signal Processing, ICASSP '99, Phoenix, AZ., 1999, pp. 1221-1224.

[25] V. Strela, "Multiwavelets: Theory and Applications " Doctor of Philosophy in Mathematics, Department of Mathematics Massachusetts Institute of Technology, 1996.

[26] M. B. Martin, "Applications of multiwavelets to image compression," M.S. thesis, Electrical Engineering Department, Virginia Polytechnic Institute and State University (Virginia Tech), USA, 1999.

[27] M. H. M. Nerma, et al., "On DT-CWT Based OFDM: PAPR Analysis," in Multi-Carrier Systems \& Solutions 2009, ed: Springer, 2009, pp. 207-217.

[28] S. Plass, et al., Multi-Carrier Systems \& Solutions 2009. Proceedings from the 7th International Workshop on Multi-Carrier Systems \& Solutions, May 2009, Herrsching, Germany vol. 41: Springer Science \& Business Media, 2009.

[29] V. Strela and A. T. Walden, "Orthogonal and biorthogonal multiwavelets for signal denoising and image compression," Orlando, FL, USA, 1998, pp. 96107 